\title{
Video Article \\ Isolation and Characterization of Cardiac Mesenchymal Stromal Cells from Endomyocardial Bioptic Samples of Arrhythmogenic Cardiomyopathy Patients
}

Chiara Assunta Pilato ${ }^{* 1,2}$, Ilaria Stadiotti ${ }^{*}$, Angela Serena Maione ${ }^{1}$, Valentina Saverio ${ }^{1}$, Valentina Catto ${ }^{3}$, Fabrizio Tundo ${ }^{3}$, Antonio Dello Russo ${ }^{3}$, Claudio Tondo ${ }^{2,3}$, Giulio Pompilio ${ }^{1,2}$, Michela Casella* ${ }^{{ }^{3}}$, Elena Sommariva* ${ }^{1^{1}}$

${ }^{1}$ Vascular Biology and Regenerative Medicine Unit, Centro Cardiologico Monzino-IRCCS

${ }^{2}$ Department of Clinical Sciences and Community Health, Università degli Studi di Milano

${ }^{3}$ Heart Rhythm Center, Centro Cardiologico Monzino-IRCCS

*These authors contributed equally

Correspondence to: Elena Sommariva at esommariva@ccfm.it

URL: https://www.jove.com/video/57263

DOI: doi: $10.3791 / 57263$

Keywords: Developmental Biology, Issue 132, Endomyocardial biopsy, Arrhythmogenic Cardiomyopathy, Cardiac Mesenchymal Stromal Cells, Adipogenesis, ARVC, isolation, characterization, differentiation.

Date Published: 2/28/2018

Citation: Pilato, C.A., Stadiotti, I., Maione, A.S., Saverio, V., Catto, V., Tundo, F., Dello Russo, A., Tondo, C., Pompilio, G., Casella, M., Sommariva, E. Isolation and Characterization of Cardiac Mesenchymal Stromal Cells from Endomyocardial Bioptic Samples of Arrhythmogenic Cardiomyopathy Patients. J. Vis. Exp. (132), e57263, doi:10.3791/57263 (2018).

\section{Abstract}

A normal adult heart is composed of several different cell types, among which cardiac mesenchymal stromal cells represent an abundant population. The isolation of these cells offers the possibility of studying their involvement in cardiac diseases, and, in addition, provides a useful primary cell model to investigate biological mechanisms.

Here, the method for the isolation of C-MSC from arrhythmogenic cardiomyopathy patients' bioptic samples is described. The endomyocardial biopsy sampling is guided in the right ventricular areas adjacent to the scar visualized by electro-anatomical mapping. The digestion of the biopsies in collagenase and their plating on a plastic dish in culture medium to allow C-MSC growth is described. The isolated cells can be expanded in culture for several passages. To confirm their mesenchymal phenotype, the description of immuno-phenotypical characterization is provided. C-MSC are able to differentiate into several cell types like adipocytes, chondrocytes, and osteoblasts: in the context of ACM, characterized by adipocyte deposits in patients' hearts, the protocols for the adipogenic differentiation of C-MSC and the characterization of lipid droplet accumulation are described.

\section{Video Link}

The video component of this article can be found at https://www.jove.com/video/57263/

\section{Introduction}

Mesenchymal Stromal Cells (MSC) are adult cells with an important supportive function in many tissues ${ }^{1}$. Bone marrow represents the historical source of MSC, but they can be isolated from different tissues including placenta, adipose tissue, cord blood, liver, and heart ${ }^{1,2}$.

In 2006, the International Society for Cellular Therapy (ISCT) specified, for the first time, the minimal criteria to define human MSC ${ }^{3}$. In particular, MSC must have the ability to adhere to plastic. They express specific surface antigens: the positivity for CD44, CD105, CD29, and CD90 mesenchymal markers, and the negativity for CD14, CD45, CD34, CD31 hematopoietic and endothelial markers characterize MSC. Due to the lack expression of HLA-DR, MSC are unable to trigger alloreactivity. Moreover, they are multipotent cells with the potential to differentiate toward adipogenic, chondrocyte, and osteoblast lineages ${ }^{1,3}$

Focusing on cardiac cellular composition, cardiac mesenchymal stromal cells (C-MSC) are abundant in a normal adult heart ${ }^{4,5}$. They play a critical role both in the normal cardiac function and pathological conditions. While, physiologically, C-MSC provide a microenvironment that supports the structural and functional integrity of the myocardium, in heart diseases they are activated in response to heart injury participating in the wound healing and fibrotic remodeling ${ }^{6,7}$.

Recently, the involvement of C-MSC in arrhythmogenic cardiomyopathy (ACM) adipose substitution has been demonstrated ${ }^{8}$. In particular, $\mathrm{ACM}$ is a genetic disorder mainly caused by mutations in desmosomal genes that lead to myocardial fibro-fatty replacement, mainly in the right ventricle $^{9}$. This substitution, extending from epicardium to endocardium, creates a non-conductive substrate that elicits progressive heart failure and worsens ventricular arrhythmias, which can lead, in severe cases, to sudden death. Sommariva et al. demonstrated the mesenchymal origin of the pre-adipocytes present in ACM patients' explanted heart sections. Furthermore, C-MSC isolated from endomyocardial biopsies of ACM hearts express desmosomal genes and therefore can be affected by their mutations. In particular, under adipogenic conditions, ACM C-MSC 
accumulate more lipids than those from control hearts. This evidence leads to the conclusion that C-MSC both have an active role in the disease pathogenesis and represent a valid cell model to study ACM.

To facilitate future research in this context or in other heart diseases for which a cardiac biopsy is indicated, a detailed protocol is presented for the isolation of C-MSC from small fragments of endomyocardial tissue, their expansion, their immuno-phenotypical characterization, and adipogenic differentiation.

\section{Protocol}

This approach complies with the Declaration of Helsinki and the collection of right ventricular samples was approved (07/06/2012) by "Centro Cardiologico Monzino IRCCS" Ethics Committee.

\section{Solutions}

1. Make TMES medium for C-MSC culture with Iscove's Modified Dulbecco's Medium (IMDM), supplemented with $20 \%$ Fetal Bovine Serum (FBS), $10 \mathrm{ng} / \mathrm{mL}$ basic fibroblast growth factor, 10,000 U/mL Penicillin, 10,000 $\mu \mathrm{g} / \mathrm{mL}$ Streptomycin, and $0.02 \mathrm{M} \mathrm{L-Glutamine.} \mathrm{Filter} \mathrm{the} \mathrm{TMES}$ medium using a $0.22 \mu \mathrm{m}$ sterile filter unit, and store at $4{ }^{\circ} \mathrm{C}$.

2. To prepare collagenase solution, resuspend the collagenase NB4 mix in IMDM medium to obtain a final solution with a concentration of 3 $\mathrm{mg} / \mathrm{mL}$. Vortex gently to dissolve the powder, and filter the collagenase solution using a $0.2 \mu \mathrm{m}$ syringe filter. Aliquot $1 \mathrm{~mL}$ volume into $2 \mathrm{~mL}$ sterile tubes and store at $-20^{\circ} \mathrm{C}$ until needed for use.

3. To prepare T. ADIPO medium, make adipogenic medium with IMDM medium supplemented with 10\% FBS, 0.5 mM 3-isobutyl-1methylxanthine (pre-diluted in dimethyl sulfoxide (DMSO) at a concentration of $0.5 \mathrm{M}$ and kept at $-20^{\circ} \mathrm{C}$ until use), $1 \mu \mathrm{M}$ hydrocortisone (prediluted in DMSO at $100 \mathrm{mM}$ and further diluted to $10 \mathrm{mM}$ in sterile distilled water and kept at $-20^{\circ} \mathrm{C}$ until use) and $0.1 \mathrm{mM}$ indomethacin (prediluted in DMSO at a concentration of $0.5 \mathrm{M}$ and kept at $-20^{\circ} \mathrm{C}$ until use). Filter the T. ADIPO medium using a $0.22 \mu \mathrm{m}$ sterile filter unit and store at $4{ }^{\circ} \mathrm{C}$.

4. Prepare washing buffer composed of phosphate-buffered saline $0.0067 \mathrm{M} \mathrm{PO}_{4}$ (PBS), ethylenediamine tetra acetic acid (EDTA) 5 mM, and $5 \%$ bovine serum albumin, and store at $4{ }^{\circ} \mathrm{C}$.

5. Prepare oil red O (ORO) working solution by dissolving $1 \%$ ORO powder in $60 \%$ isopropanol, mixing for $2 \mathrm{~h}$, and filtering using a $0.22 \mu \mathrm{m}$ filter unit to remove precipitates. Store the working solution at room temperature (RT).

\section{Isolation of Cardiac Mesenchymal Stromal Cells}

\section{Cardiac biopsy collection and processing}

Note: Before starting, make sure to have the collagenase solution and the TMES medium ready.

1. Put the ACM endomyocardial biopsy (usually about $5 \mathrm{mg}$ of tissue) in a sterile tube filled with TMES and transport it to the laboratory. Note: ACM biopsies are collected in the electrophysiology operating room, according to previous reports ${ }^{10}$. Briefly, the integration of electro-anatomical mapping and intracardiac echocardiography in the right ventricle (see Video 1) is used to guide the endomyocardial biopsy (see Video 2, fluoroscopy) in correspondence to the border zone of the diseased myocardium. Healthy Control (HC) samples are supplied by a tissue bio-bank, obtained from the right ventricular free wall of cadaveric donors within $24 \mathrm{~h}$ of death (accidental death, healthy subjects). During transport and prior to dissection, store the biopsy in TMES at $4{ }^{\circ} \mathrm{C}$. Limit the time between biopsy collection and tissue processing (max $24 \mathrm{~h}$ ).

2. Set up the biological safety cabinet with required solutions to carry out the procedure up to the enzymatic digestion.

3. Put the sterilizer under the hood and turn on the instrument $15 \mathrm{~min}$ before use until the temperature rises $200-250{ }^{\circ} \mathrm{C}$.

4. Sterilize scissors and tweezers for $10 \mathrm{~s}$. Make sure the sterilized instruments are cold before use. Prepare disposable sterile scalpels

5. Place the tubes with the biopsy in the sterile hood. Open the tube and transfer the biopsy into a sterile plate.

6. Wash the right ventricle biopsy twice with $3 \mathrm{~mL}$ of sterile PBS. If you want take a picture of the biopsy at the microscope.

7. Transfer the right ventricle biopsy, using sterile tweezers, into a sterile $2 \mathrm{~mL}$ tube containing $1 \mathrm{~mL}$ of the collagenase solution.

8. Cut the sample into $0.5-1 \mathrm{~mm}^{3}$ pieces with sterile scissors.

9. Incubate for $1.5 \mathrm{~h}$ at $37^{\circ} \mathrm{C}$ under continuous rotating agitation.

10. Centrifuge the digested solution at $400 \times \mathrm{g}$ for $10 \mathrm{~min}$ at RT.

11. Remove the supernatant and add $1 \mathrm{~mL}$ of sterile PBS to wash the pellet.

12. Centrifuge at $400 \times \mathrm{g}$ for $10 \mathrm{~min}$ at RT.

13. Remove the supernatant and resuspend the pellet in $1 \mathrm{~mL}$ of TMES medium.

14. Plate the obtained suspension onto sterile $60-\mathrm{mm}$ tissue culture treated plate and add TMES medium to the final volume of $3 \mathrm{~mL}$. Incubate the plate in a cell culture incubator at $37^{\circ} \mathrm{C}$ with $5 \% \mathrm{CO}_{2}$.

Note: A higher volume of medium may prevent the correct adhesion of a digested biopsy fragments.

15. After $24 \mathrm{~h}$, discard the medium to remove non-adherent cells and debris.

Note: Single dissociated mesenchymal cells can attach to the plastic surface and give rise to clones; also, undigested small biopsy fragments can attach to the plastic dish and allow cell sprouting.

16. Wash the plate twice with $5 \mathrm{~mL}$ of sterile PBS, and add $3 \mathrm{~mL}$ of fresh TMES medium.

17. Replace the TMES medium three times a week until the cells are $80 \%$ confluent.

Note: The number of attached cells depends on the quality, amount of tissue, and on the efficiency of digestion.

\section{Cell Expansion}

Note: Before starting make sure to have TMES medium ready. 
1. When the cells are $80 \%$ confluent, transfer the digested small bioptic samples into a new sterile 60 -mm tissue culture treated plate, and add 3 $\mathrm{mL}$ of fresh TMES medium.

Note: The bioptic samples can be re-used for further C-MSC isolation. It is possible to repeat this procedure until there is a significant number of isolated cells.

2. Wash the attached cells twice with $3 \mathrm{~mL}$ of sterile PBS. Add trypsin-EDTA solution $\left(0.5 \mathrm{~mL}\right.$ for a $60-\mathrm{mm}$ dish), and incubate at $37{ }^{\circ} \mathrm{C}$ for $3-5$ min to allow cell detachment.

3. When the cells are detached from the surface of the plate, add $0.5 \mathrm{~mL}$ of sterile FBS to inactivate the trypsin and collect cells into a new 50 $\mathrm{mL}$ sterile tube.

4. Wash the plate twice with $5 \mathrm{~mL}$ of sterile PBS to add the remaining cells to the same tube.

5. Centrifuge the cells at $400 \times \mathrm{g}$ for $10 \mathrm{~min}$ at RT.

6. Remove the supernatant and resuspend the pellet in $1 \mathrm{~mL}$ of TMES medium.

7. Load $10 \mu \mathrm{L}$ of the cell suspension in the cell counting chamber

Note: If the cells are too concentrated, dilute the initial suspension 1:10 in PBS and load $10 \mu \mathrm{L}$ of the diluted cells in the cell counting chamber.

8. Under the microscope, count cells in 3 large squares and on the lines of two of their sides and calculate the average number of cells. Note: To obtain the number of cells $/ \mathrm{mL}$, the average of counted cells is multiplied by $10^{4}$, the dilution factor of the cell counting chamber. If the cells have been further diluted, multiply for the dilution factor. The ratio between the cell number to seed and the cell concentration (number of cells $/ \mathrm{mL}$ ) corresponds to the volume (in $\mathrm{mL}$ ) of initial suspension to be plated for the next step (point 3.9).

9. Plate the resuspension onto sterile $100-\mathrm{mm}$ tissue culture treated plates at a final concentration of $5,000-10,000 \mathrm{cells} / \mathrm{cm}^{2}$ and add TMES medium to the final volume of $8 \mathrm{~mL}$. Incubate the plate in a cell culture incubator.

Note: Repeat the cell expansion procedure when the cells are $70-80 \%$ confluent. At every passage $(\mathrm{P})$, it is recommended to cryopreserve part of the cells and plate the remaining amount. Expand the cells until P3 or P4 and use for fluorescent-activated cell sorter (FACS) analysis (section 4) and adipogenic differentiation (section 5).

10. For the cryopreservation after detachment, centrifuge the cells at $400 \times \mathrm{g}$ for $10 \mathrm{~min}$ at RT.

11. Count cells as described (steps $3.7-3.8$ ) and resuspend $1 \times 10^{6}$ cells in $900 \mu \mathrm{L}$ of sterile FBS. Transfer to a sterile cryo-vial and add $100 \mu \mathrm{L}$ of sterile DMSO and mix.

12. Quickly store the cryo-vials into a freezing container at $-80^{\circ} \mathrm{C}$ for at least 2 days and then transfer the vials into liquid nitrogen.

\section{Characterization of Cardiac Mesenchymal Stromal Cells by Flow Cytometry}

Note: Before starting, make sure to have the cell dissociation reagent, washing buffer, and the specific antibodies prepared.

1. When the cell number reaches at least $3 \times 10^{6}$ (count the cells as described in $3.7-3.8$ ), wash the 100 -mm plate twice with $10 \mathrm{~mL}$ of sterile PBS.

2. Add $5 \mathrm{~mL}$ of a cell dissociation reagent and wait $7-10 \mathrm{~min}$ at $\mathrm{RT}$ to allow the cell detachment.

3. When the cells are detached from the surface of the plate, add $15 \mathrm{~mL}$ of sterile washing buffer and collect the suspension into a new $50 \mathrm{~mL}$ sterile tube.

4. Wash the plate twice with $10 \mathrm{~mL}$ of washing buffer and add the remaining cells to the same tube.

5. Centrifuge the cells at $400 \times \mathrm{g}$ for $10 \mathrm{~min}$ at RT and resuspend the pellet in $1 \mathrm{~mL}$ of washing buffer.

6. Count the cells as described (steps $3.7-3.8$ ), and transfer $3 \times 10^{6}$ cells into a new sterile tube and add washing buffer to the final volume of $1.5 \mathrm{~mL}$.

Note: The total cell number and the total volume of washing buffer depends on the number of selected markers used for the analysis $\left(3 \times 10^{5}\right.$ cells in $100 \mu \mathrm{L}$ for each FACS polystyrene tube).

7. Distribute $100 \mu \mathrm{L}$ of cellular suspension in 12 different FACS polystyrene tubes, and add the specific antibody at the concentration indicated in the product datasheet.

Note: Antibodies used for C-MSC characterization are CD34, CD105, CD45, CD29, CD90, CD44, CD31, CD14, and HLA-DR (Table 1). It is important to prepare a control sample composed of $100 \mu \mathrm{L}$ of resuspended cells stained with the isotype control to check the specificity of the signal.

8. Incubate the samples for $15 \mathrm{~min}$ in the dark.

9. Add $1 \mathrm{~mL}$ of sterile washing buffer to each tube to stop the reaction.

10. Centrifuge the cells at $400 \times \mathrm{g}$ for $10 \mathrm{~min}$ at RT.

11. Remove the supernatant and resuspend the pellet in $250 \mu \mathrm{L}$ of washing buffer.

12. Proceed to C-MSC characterization with flow cytometry.

\section{Adipogenic Differentiation of Cardiac Mesenchymal Stromal Cells}

Note: Before starting, make sure to have prepared T. ADIPO medium and ORO working solution.

1. Culture in T. ADIPO medium

1. Detach and count the cells as described in section 3.

2. Plate $3 \times 10^{5}$ cells onto each well of a sterile 6 -well tissue culture treated plate in $2 \mathrm{~mL}$ of T. ADIPO medium.

3. Let C-MSC differentiate in T. ADIPO medium for $72 \mathrm{~h}$ or 1 week, changing medium every $2-3$ days.

2. Oil Red O staining

Note: Use Oil Red O (ORO) staining to test the lipid accumulation in C-MSC.

1. Place the 6-well tissue culture plateunder the fume hood.

2. Remove the adipogenic medium and wash the cells twice with $2 \mathrm{~mL}$ of PBS.

3. Add the enough volume of $4 \%$ paraformaldehyde (PFA) to cover each well. Fix the cells for 5 min at RT.

4. Discard PFA and wash the cells twice with $2 \mathrm{~mL}$ of PBS. 
5. Incubate the fixed cells with ORO working solution for $1 \mathrm{~h}$ at RT, using enough volume to cover each well.

Note: Do not keep the 6-well tissue culture plate under the fume hood during ORO incubation to avoid the evaporation of ORO working solution.

6. Remove all ORO working solution and wash with $2 \mathrm{~mL}$ of PBS 3 - 5 times until the plate is completely cleaned; discard washes. Stop the washes when the PBS used is clear and when you don't see, under the microscope, unspecific staining out of the cells.

7. Capture 20 images at 20X magnification for each well using the inverted tissue culture phase-contrast microscope.

8. Open the pictures with the image processing program to quantify the cell ORO accumulation.

9. Separate the different color channels through the "split channels" function in order to quantify only the luminance in the 255-red channel.

10. Count the number of cells for each picture by counting the nuclei. Normalize the ORO signal intensity on the cell number.

11. Calculate the average of the results obtained for each picture of the same sample.

\section{Representative Results}

Cardiac stromal cells isolation: The C-MSC isolation from endomyocardial biopsies procedure is summarized in Figure 1.

Cardiac stromal cells mesenchymal characterization: As established by the International Society for Cellular Therapy (ISCT), the minimal criteria for defining multipotent mesenchymal stromal cells includes their immuno-phenotypic characterization ${ }^{3}$. In particular, to confirm their mesenchymal lineage, cells are incubated with appropriate FITC/PE/APC-conjugated antibodies and analyzed by flow cytometry. All of the antibodies used in the C-MSC characterization are listed in Table of Materials.

As illustrated in Figure 2, C-MSC obtained from biopsies are positive for the specific mesenchymal surface antigens CD29, CD44, and CD105. The percentage of CD90 positive cells is variable, as reported previously ${ }^{11}$. The endothelial (CD31, CD34), monocyte/macrophage (CD14), hematopoietic (CD45) markers, and major histocompatibility complex (HLA-DR) are not expressed (Figure 2).

Cardiac mesenchymal stromal cells adipogenic differentiation: To prompt their adipogenic differentiation, C-MSC obtained from patients affected by ACM and HC have to be cultured in T. ADIPO medium (see Solutions section). The cells are maintained in culture for $72 \mathrm{~h}$ or 1 week, replacing the medium twice a week.

The accumulation of intracellular lipid droplets is evidenced by ORO staining (Figure 3). Differences in the ability, as well as in the degree of differentiation, can be observed between the cells obtained from ACM and HC. As shown in the representative images, ACM C-MSC accumulates more lipid droplets than HC C-MSC after $72 \mathrm{~h}$ of culture in adipogenic medium (Figure 3). These differences are maintained also when the cells are exposed to adipogenic differentiation conditions for a longer period (1 week) (Figure 3).

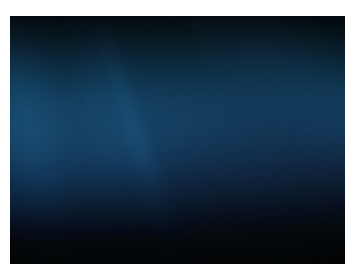

Video 1: Integration of electroanatomical map and intracardiac echocardiography. Endocardial unipolar electroanatomical maps of the right ventricle (left anterior oblique and right anterior oblique views) in a patient who undergoes endomyocardial biopsy (lower panels). Limited areas of low voltage (red/green) are visible at the apex. Endomyocardial bioptic samples (tagged as circle) are collected in correspondence to the diseased myocardium and the interventricular septum. Real-time intracardiac echocardiography allows checking the correct position of the bioptome at the target area (upper panel). Please click here to view this video. (Right-click to download.)

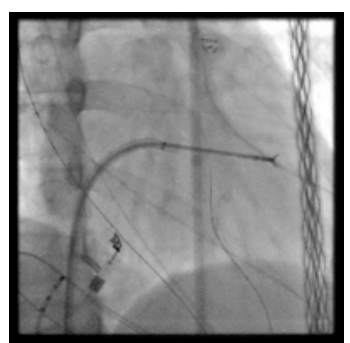

Video 2: Fluoroscopy video in right anterior oblique view. The bioptome is inserted through a long-deflectable sheath and advanced into the ventricle. After a careful check of the bioptome contact with the myocardium, the jaws are opened and then firmly closed to collect the sample. Please click here to view this video. (Right-click to download.) 

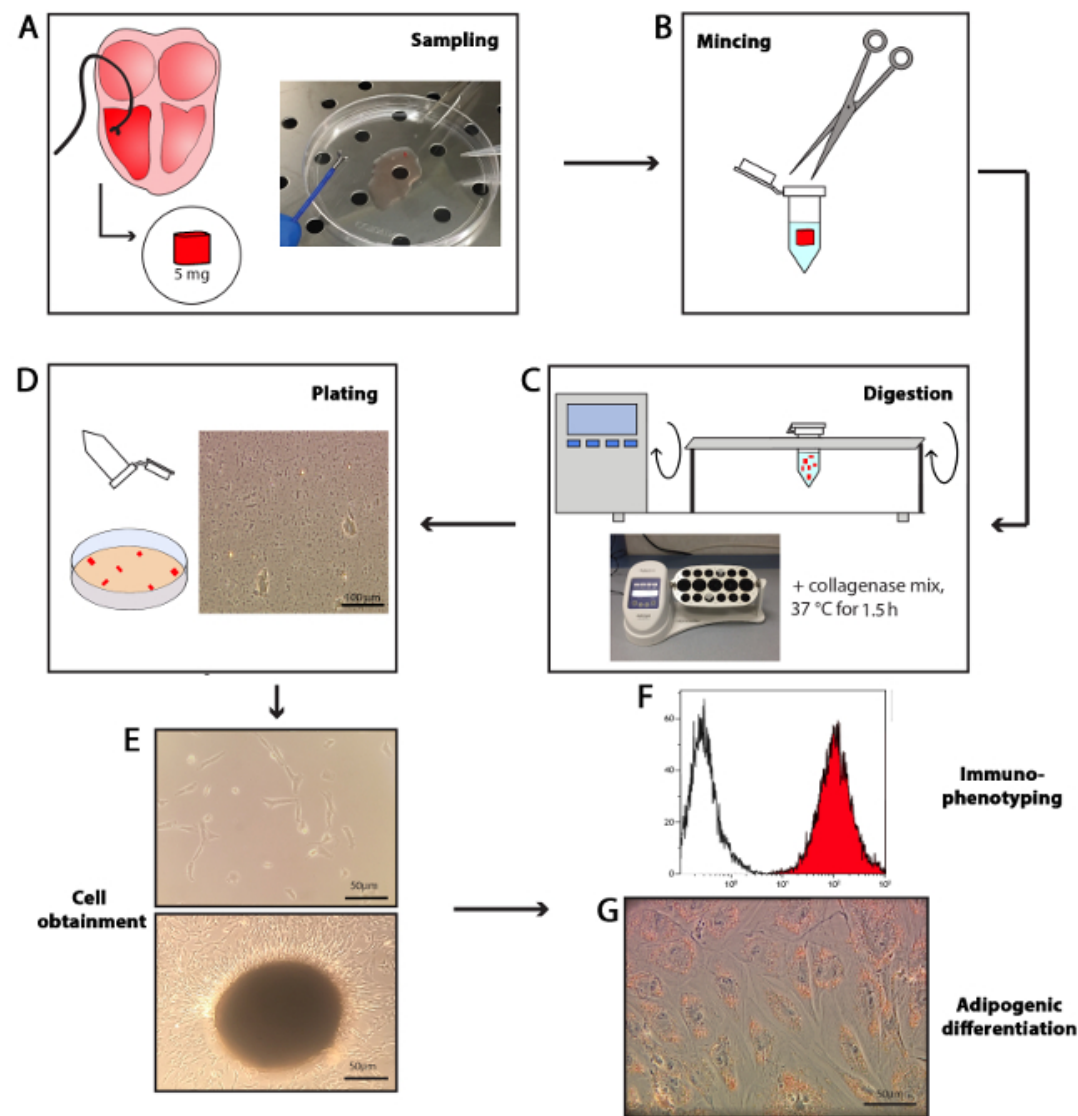

Figure 1: Procedure draft. The endomyocardial bioptic sample is collected using a bioptome catheter, a small pincer-shaped cutting instrument. The weight of the obtained biopsy is approximately $5 \mathrm{mg}(\mathbf{A})$. The endomyocardial bioptic sample is minced into $0.5-1 \mathrm{~mm}$ fragments, with sterile scissors (B), and collagenase solution is added. The sample is placed in a $37^{\circ} \mathrm{C}$ incubator on a rotating platform mixer for 1.5 $\mathrm{h}$ of digestion (C). The digested solution is centrifuged and plated in TMES in a plastic dish (D). C-MSC are obtained thanks to their plastic adherence properties either in single cells or clones, or sprouting from small undigested bioptic fragments (E). C-MSC are then characterized by flow cytometry $(\mathbf{F})$. C-MSC are plated in adipogenic medium and lipid droplet accumulation is tested by Oil Red O staining (G). Scale bars indicate $50 \mu \mathrm{m}$. Please click here to view a larger version of this figure.
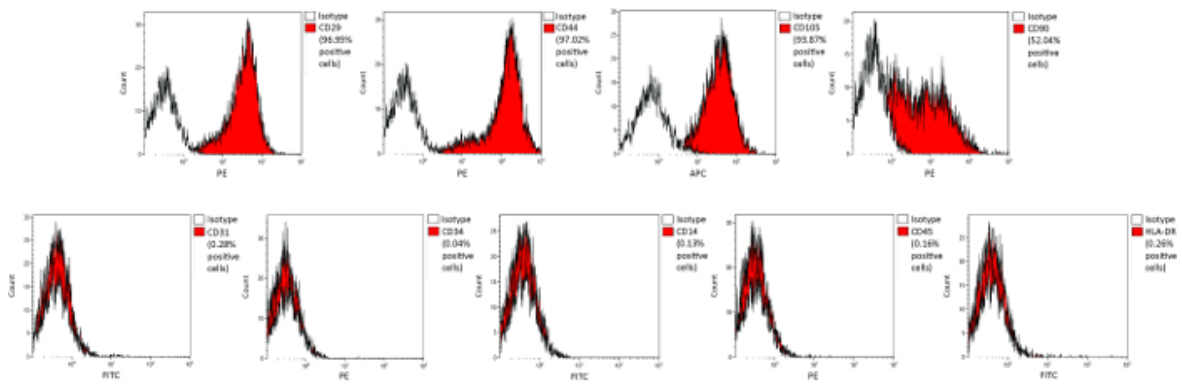

Figure 2: Representative cyto-fluorimetric profile of C-MSC. Each histogram shows the cell count vs. the intensity of fluorescence in the indicated channel (PE: phycoerythrin; APC: allophycocyanin; FITC: fluorescein-isothyocyanate). In each graph the isotype control (white) and a sample conjugated with the specific cell surface marker antibody (red) are shown. C-MSC are positive for the mesenchymal surface antigens CD29, CD44, CD105 and, partially, for CD90, whereas they do not express CD31, CD34, CD14, CD45, and HLA-DR. Please click here to view a larger version of this figure. 

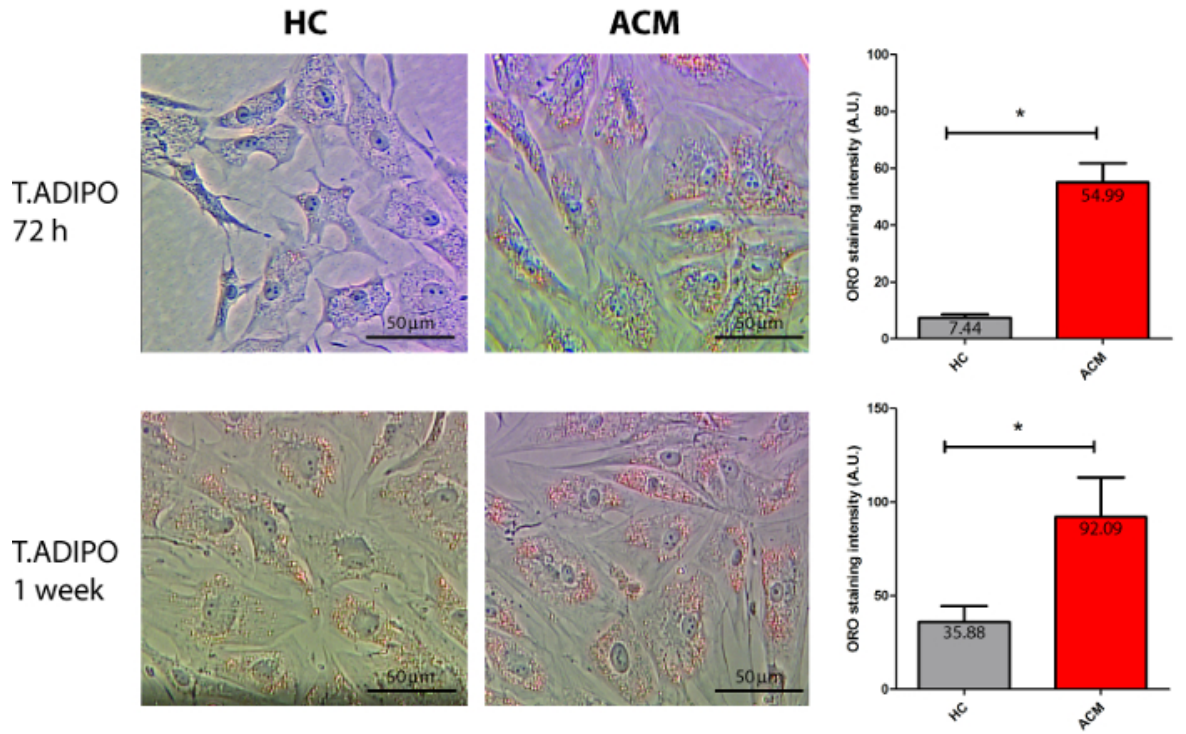

Figure 3: Adipogenic differentiation of C-MSC. Representative images of ACM and HC C-MSC, cultured in adipogenic medium for $72 \mathrm{~h}$ and 1 week, stained with ORO (left pictures; $n=3$ ). In the right graphs, quantification of the luminance of the 255-red channel staining is reported: intensity is expressed in arbitrary units (A.U.). ACM C-MSC accumulate more lipid droplets than controls. Student's T-test was used, ${ }^{*} p<0.05$. Scale bars indicate $50 \mu \mathrm{m}$. Please click here to view a larger version of this figure.

\section{Discussion}

MSC and C-MSC: MSC are multipotent cells resident in the stromal fraction of different adult tissues, such as bone marrow, adipose tissue, cartilage, brain, skin, fetal annexes, and heart ${ }^{12}$. Different studies have been performed to isolate and characterize them for potential applications in basic and translational research ${ }^{12,13}$.

In healthy conditions, MSC are quiescent, self-renewing at low rates ${ }^{14}$. Since they are exposed to environmental pathological changes, they react fostering tissue remodeling through either direct transdifferentiation, matrix deposition, or the paracrine effect ${ }^{14}$.

The cardiac MSC (C-MSC) represent a large non-myocyte cell population of the heart ${ }^{4}$. They originate from the epicardium and migrate into the myocardium undergoing the process of epithelial-to-mesenchymal transition ${ }^{15}$. They contribute to the mechanical and electrical integrity of the cardiac structure, both in physiological and in pathological states, through interactions with cardiomyocytes and extracellular matrix homeostasis $^{7,16}$. However, the broad range of C-MSC functions is still not completely understood. A deeper knowledge of their role both in physiological and pathological conditions can be facilitated by in vitro studies performed after their isolation.

C-MSC have been obtained from different districts of the human heart, such as the atrial appendage $e^{2,17}$ and right ventricle ${ }^{18}$.

Recently, C-MSC from human right ventricular endomyocardial bioptic samples have been obtained ${ }^{8}$, demonstrating that the source tissue could be as little as $3-5 \mathrm{mg}$.

Possible applications: The method outlined in this manuscript allows obtaining cells with few simple passages, such as digestion and selection for plastic adherence, from very small heart specimens.

C-MSC can be considered a cell model, since they are easy to amplify and maintain in vitro, and are able to differentiate into cells of mesenchymal lineage (endothelium, osteocytes, and adipocytes). Moreover, the possibility of obtaining cells directly from patients constitutes a great in vitro tool for mechanistic studies in the context of personalized/precision medicine. Indeed, these cells carry the genetic background and eventually specific mutations of the donors, and are influenced by the specific patients' characteristics, such as clinical conditions, age, sex, lifestyle, and medications. Moreover, the possibility of sorting them for different markers may allow the study of specific C-MSC subsets ${ }^{19}$.

C-MSC are known to be active players in different cardiovascular diseases, mostly characterized by adverse remodeling of the heart. Therefore, they represent candidate targets for novel therapeutic strategies to counteract heart diseases ${ }^{8,20}$.

C-MSC stem-like properties and their lack of significant immunogenicity suggests their potential application in cell-therapy for cardiac regenerative medicine. Indeed, like MSC from bone marrow or other sources, C-MSC could be potentially used both in autologous and in allogenic settings, without the need for matching between donor and recipient ${ }^{21}$.

Moreover, C-MSC, being isolated directly from heart tissue, have the advantage of being preconditioned by the cardiac micro-environment and epigenetic profile. In the context of cardiac regenerative medicine, this could be particularly important to obtain successful results.

To date, preclinical studies of regenerative medicine identified useful therapeutic potential in the C-MSC and their paracrine activity ${ }^{18,22,23}$. Importantly, clinical trials in which the cell source is the heart are underway either with cardiosfere-derived cells or with subpopulations of CMSC $^{13,24,25}$. However, as for bone-marrow-derived MSC, different protocols may be necessary to obtain clinical grade C-MSC ${ }^{26}$ 
C-MSC in ACM: The presented protocol is mostly suitable for the study of pathologies for which an endocardial biopsy is indicated. ACM patients undergo bioptic procedures for diagnostic purpose ${ }^{27}$. Their myocardium is gradually substituted by scar-tissue, an electrically inert tissue composed of adipocytes and fibrosis. In order to guide the bioptic sampling to the scar area, where the diagnostic yield is maximal, endomyocardial mapping is used ${ }^{10,28,29}$. The samples used in this protocol are taken in the border zone of the diseased myocardium.

Sommariva et al. has recently defined a pivotal role of C-MSC in the pathogenesis of $\mathrm{ACM}^{8}$, demonstrating that C-MSC are active players in ACM heart adipogenesis, since preadipocytes in those hearts are of mesenchymal origin. Moreover, C-MSC isolated with the present protocol from ACM patients' biopsies showed more propensity to both lipid accumulation and adipogenesis than controls. For this reason, these cells could be used to confirm some of the molecular mechanisms of ACM, proving their suitability as a cell model for mechanistic studies ${ }^{9}$.

Limitations and critical steps: Despite the advantages of obtaining C-MSC directly from patients (see the paragraph "Possible applications"), this protocol is subjected to different limitations.

First of all, the cardiac bioptic procedure is invasive and often avoided if not strictly necessary. Indeed, sampling cardiac tissue is both ethically and technically problematic. Reasons for performing a cardiac biopsy may be the achievement of a definite diagnosis in the context of cardiomyopathies in differential diagnosis, monitoring the status of cardiac transplants, or ascertaining the presence of a heart tumor ${ }^{30}$. Therefore, only patients for which an endomyocardial biopsy is indicated by consensus statement ${ }^{31}$ can be enrolled for research on C-MSC. Moreover, the cardiac bioptic procedure can have clinical complications, above all in cardiomyopathic hearts. Therefore, electrophysiologist's samplings are always cautious and bioptic samples could be very small, compromising the isolation of cells. Future experiments could overcome this issue by tuning collagenase concentration or timing of digestion.

C-MSC, as all primary human cells, show a high variability among different subjects in all phenotypes. Indeed, cells from different subjects are not only genetically different, but also subjected to variable environmental conditioning. Specifically, within this experiment, a high variability in cell isolation, growth, and adipogenic differentiation is observed.

Critical steps of the present protocol have to be acknowledged. If the bioptic sample includes capillaries, they must be removed to avoid the parallel isolation of endothelial cells, which may contaminate the C-MSC culture, and can be evidenced by the FACS analysis (positivity for CD31). To obtain an efficient adipogenic differentiation, cells must be in an active growth phase. The degree of confluence may also influence lipid accumulation.

Significance of the method: With respect to previous methods of isolation of mesenchymal stromal cells, this is the first time where the description of C-MSC obtainment directly from human ventricular bioptic samples is proposed in detail. Although this method is suggested for the processing of ACM patient samples, it is potentially applicable to all the patients for which a cardiac biopsy is indicated.

This protocol represents a useful implementation of previous methods for the obtainment of cells that required bigger cardiac samples ${ }^{32}$, which are often difficult to collect.

Moreover, the sample source constitutes an interesting innovation. While the ventricular biopsy is usually performed on the septum ${ }^{33}$, this protocol takes into account samples obtained from the right ventricular free wall. Cells derived from the diseased right ventricular district may be more representative of the pathologic status of diseases involving RV.

In addition, some of the reagents used in the present protocol are different with respect to other C-MSC isolation and differentiation methods ${ }^{32}$. For example, the type of collagenase proposed in this manuscript is a mix of class I and class II collagenases with a balanced ratio of proteolytic activities. Moreover, the digestion solution is composed of the collagenase mix dissolved in the same basal medium (IMDM) used for the preparation of C-MSC culture medium, allowing isolated C-MSC to adapt to future growth conditions.

In addition, though sorting procedures could standardize the cell batch, using the whole C-MSC population, isolated only through the plastic adherence property of these cells, constitutes a simplification without altering the immunophenotypic characteristics of C-MSC. The composition of T. ADIPO proposed in this manuscript is able to lead to adipogenic differentiation, avoiding the metabolic dysregulation induced by other components such as insulin.

Moreover, the proposed method of lipid accumulation quantification, which is based on the evaluation of the ORO colorimetric intensity, provides more information about the quantity of the accumulated lipids, if compared with methods based only on the percentage of cells positive to the ORO staining. Often lipid accumulation is quantified by extracting the ORO incorporated by cells with isopropanol and measuring its absorbance. However, this method requires more passages and is subjected to variability due to isopropanol evaporation.

\section{Disclosures}

The authors have nothing to disclose.

\section{Acknowledgements}

This work was funded by Italian Ministry of Health, Ricerca Corrente to Centro Cardiologico Monzino-IRCCS.

\section{References}

1. Brooke, G. et al. Therapeutic applications of mesenchymal stromal cells. Semin Cell Dev Biol. 18 (6), 846-858 (2007).

2. Rossini, A. et al. Human cardiac and bone marrow stromal cells exhibit distinctive properties related to their origin. Cardiovasc Res. 89 (3), 650-660 (2010). 
3. Dominici, M. et al. Minimal criteria for defining multipotent mesenchymal stromal cells. The International Society for Cellular Therapy position statement. Cytotherapy. 8 (4), 315-317 (2006).

4. Doppler, S. A. et al. Cardiac fibroblasts: more than mechanical support. J Thorac Dis. 9 (Suppl 1), S36-S51 (2017).

5. Moore-Morris, T., Guimaraes-Camboa, N., Yutzey, K. E., Puceat, M., \& Evans, S. M. Cardiac fibroblasts: from development to heart failure. $J$ Mol Med (Berl). 93 (8), 823-830 (2015).

6. Jugdutt, B. I. Ventricular remodeling after infarction and the extracellular collagen matrix: when is enough enough? Circulation. 108 (11), 1395-1403 (2003).

7. Brown, R. D., Ambler, S. K., Mitchell, M. D., \& Long, C. S. The cardiac fibroblast: therapeutic target in myocardial remodeling and failure. Annu Rev Pharmacol Toxicol. 45 657-687 (2005).

8. Sommariva, E. et al. Cardiac mesenchymal stromal cells are a source of adipocytes in arrhythmogenic cardiomyopathy. Eur Heart J. 37 (23), 1835-1846 (2016).

9. Sommariva, E., Stadiotti, I., Perrucci, G. L., Tondo, C., \& Pompilio, G. Cell models of arrhythmogenic cardiomyopathy: advances and opportunities. Dis Model Mech. 10 (7), 823-835 (2017).

10. Casella, M. et al. Electroanatomical mapping systems and intracardiac echo integration for guided endomyocardial biopsy. Expert Rev Med Devices. 14 (8), 609-619 (2017).

11. Beltrami, A. P. et al. Multipotent cells can be generated in vitro from several adult human organs (heart, liver, and bone marrow). Blood. 110 (9), 3438-3446 (2007).

12. Silva Meirelles, L., Chagastelles, P. C., \& Nardi, N. B. Mesenchymal stem cells reside in virtually all post-natal organs and tissues. J Cell Sci. 119 (Pt 11), 2204-2213 (2006).

13. Cencioni, C. et al. The double life of cardiac mesenchymal cells: Epimetabolic sensors and therapeutic assets for heart regeneration. Pharmacol Ther. 171 43-55 (2017).

14. Caplan, A. I. Mesenchymal stem cells. J Orthop Res. 9 (5), 641-650 (1991).

15. Germani, A., Foglio, E., Capogrossi, M. C., Russo, M. A., \& Limana, F. Generation of cardiac progenitor cells through epicardial to mesenchymal transition. J Mol Med (Berl). 93 (7), 735-748 (2015).

16. Souders, C. A., Bowers, S. L., \& Baudino, T. A. Cardiac fibroblast: the renaissance cell. Circ Res. 105 (12), 1164-1176 (2009).

17. Di Maggio, S. et al. Non-oxidizable HMGB1 induces cardiac fibroblasts migration via CXCR4 in a CXCL12-independent manner and worsens tissue remodeling after myocardial infarction. Biochim Biophys Acta. (2017).

18. Czapla, J. et al. Human Cardiac Mesenchymal Stromal Cells with CD105+CD34- Phenotype Enhance the Function of Post-Infarction Heart in Mice. PLoS One. 11 (7), e0158745 (2016).

19. Gambini, E. et al. C-kit+ cardiac progenitors exhibit mesenchymal markers and preferential cardiovascular commitment. Cardiovasc Res. 89 (2), 362-373 (2010).

20. Gourdie, R. G., Dimmeler, S., \& Kohl, P. Novel therapeutic strategies targeting fibroblasts and fibrosis in heart disease. Nat Rev Drug Discov. 15 (9), 620-638 (2016).

21. Le Blanc, K., Tammik, C., Rosendahl, K., Zetterberg, E., \& Ringden, O. HLA expression and immunologic properties of differentiated and undifferentiated mesenchymal stem cells. Exp Hematol. 31 (10), 890-896 (2003).

22. Detert, S. et al. The Atrial Appendage as a Suitable Source to Generate Cardiac-derived Adherent Proliferating Cells for Regenerative Cellbased Therapies. J Tissue Eng Regen Med. (2017).

23. Miteva, K. et al. Human cardiac-derived adherent proliferating cells reduce murine acute Coxsackievirus B3-induced myocarditis. PLoS One. 6 (12), e28513 (2011).

24. Bassetti, B., Capogrossi, M. C., \& Pompilio, G. Power Is Nothing Without Control: The Enduring Search for the Best Cell in Cardiac Cell Therapy at a Crossroads. Circ Res. 119 (9), 988-991 (2016).

25. Nigro, P. et al. Cell therapy for heart disease after 15 years: Unmet expectations. Pharmacol Res. (2017).

26. Ikebe, C., \& Suzuki, K. Mesenchymal stem cells for regenerative therapy: optimization of cell preparation protocols. Biomed Res Int. 2014 $951512(2014)$.

27. Marcus, F. I. et al. Diagnosis of arrhythmogenic right ventricular cardiomyopathy/dysplasia: proposed modification of the task force criteria. Circulation. 121 (13), 1533-1541 (2010).

28. Pieroni, M. et al. High prevalence of myocarditis mimicking arrhythmogenic right ventricular cardiomyopathy differential diagnosis by electroanatomic mapping-guided endomyocardial biopsy. J Am Coll Cardiol. 53 (8), 681-689 (2009).

29. Casella, M. et al. Feasibility of combined unipolar and bipolar voltage maps to improve sensitivity of endomyocardial biopsy. Circ Arrhythm Electrophysiol. 8 (3), 625-632 (2015).

30. Cooper, L. T. et al. The role of endomyocardial biopsy in the management of cardiovascular disease: a scientific statement from the American Heart Association, the American College of Cardiology, and the European Society of Cardiology Endorsed by the Heart Failure Society of America and the Heart Failure Association of the European Society of Cardiology. Eur Heart J. 28 (24), 3076-3093 (2007).

31. Leone, O. et al. 2011 consensus statement on endomyocardial biopsy from the Association for European Cardiovascular Pathology and the Society for Cardiovascular Pathology. Cardiovasc Pathol. 21 (4), 245-274 (2011).

32. Monsanto, M. M. et al. Concurrent Isolation of 3 Distinct Cardiac Stem Cell Populations From a Single Human Heart Biopsy. Circ Res. 121 (2), 113-124 (2017).

33. From, A. M., Maleszewski, J. J., \& Rihal, C. S. Current status of endomyocardial biopsy. Mayo Clin Proc. 86 (11), $1095-1102$ (2011). 